\title{
The Internal Fluid Mechanics of Explosive Trace Detectors using Computational Fluid Dynamics
}

\author{
Matthew E. Staymates ${ }^{1}$, 301-975-3913, matthew.staymates@nist.gov \\ Wayne J. Smith², 703-918-4369, wayne.smith@ddlomni.com \\ Greg Gillen', 301-975-2190, greg.gillen@nist.gov \\ ${ }^{1}$ National Institute of Standards and Technology Gaithersburg, MD 20899 \\ ${ }^{2}$ DDL OMNI Engineering LLC, McLean, VA 22012.
}

\begin{abstract}
Efforts are underway in the Surface and Microanalysis Science Division at the National Institute of Standards and Technology to study the vapor transport mechanisms inside explosive trace detection instruments $(E T D \text { 's })^{1}$ and produce standard test materials to verify their performance. In most swipe-based ETD's, a woven cloth is swiped across a surface to collect micrometer-sized particles from explosive contamination. The swipe is then introduced into a thermal desorption unit where it is rapidly heated to produce an explosive aerosol or vapor. This vapor is transported to a chemical detector, typically an ion mobility spectrometer, for analysis.

Understanding the underlying physics of the flow fields within these instruments allows researchers to design better test materials for calibration and verification. In this work, several ETD thermal desorption units are modeled using computational fluid dynamics (CFD). With CFD, the governing equations of fluid motion are solved numerically for a given model geometry and boundary conditions. CFD allows one to visualize and animate flow patterns, streamlines, and recirculation zones, and reveals how vapor is transported from the surface of a swipe to the chemical analyzer. The flow-fields inside these complex geometries would otherwise be difficult, if not impossible, to observe with traditional experimental flow visualization techniques.

The thermal desorption units presented here have geometries representative of what is used in ETD's today. Results suggest that the transport efficiency of desorbed explosives can be optimized if appropriate screening procedures are followed. Issues such as velocity magnitude, pressure differential, transient effects, and buoyancy effects will be discussed.
\end{abstract}

\footnotetext{
${ }^{1}$ Certain commercial equipment, instruments, or materials are identified in this document. Such identification does not imply recommendation or endorsement by the National Institute of Standards and Technology, nor does it imply that the products identified are necessarily the best available for the purpose.
}

\section{INTRODUCTION}

In general, there are two types of trace explosives screening methods: portal-based systems, which aerodynamically sample the air around a human subject, and swipe-based instruments which rely on the manual collection of explosive residue from laptops, purses, and other small carry-on items [1]. In swipe-based sampling, a swab of cloth or other woven material is swiped over a surface to gather trace amounts of explosive material [2], and then heated in a thermal desorption unit to generate a vapor for chemical analysis. Ion mobility spectrometry (IMS) is typically the primary method of chemical analysis in explosive trace detectors (ETD's) [3]. The desorber and the associated chemical analyzer effectively comprise an ETD.

The National Institute of Standards and Technology's (NIST) Surface and Microanalysis Science Division, along with the Office of Law Enforcement Standards are working with the US Department of Homeland Security and the Transportation Security Laboratory to provide tools for fundamental metrology of explosives screening, create standardized testing methods and materials for ETD's, improve current security screening technology, and develop next-generation screening technology. This paper focuses on a new application of the recognized technique within the fluid mechanics community known as computational fluid dynamics (CFD). Fluid mechanics and its applications to Homeland Security have been recognized as playing a key role in the advancement of new technologies [4]. Here, CFD is used to investigate the internal and external flow patterns of explosive trace detection instrumentation and identify the underlying transport physics of desorbed explosives once they have been vaporized from a surface.

CFD not only assists in understanding how trace detectors work fundamentally, but it also serves as a guide for developing functional standard test materials to validate the performance of these instruments. As an example, one aspect of NIST's program in trace explosive standards uses 
inkjet printing technology to deposit known amounts of explosive solutions onto test swabs to be used as standards for swipe-based ETD's [5]. NIST researchers use the results of CFD simulations to understand where the explosive deposit should be located on the swab to maximize vapor transport to the detector.

This paper will describe the computational results of six possible desorber configurations that represent typical implementations used in ETD's. Specific dimensions and parameters of the models are withheld, as they are not necessary in understanding the general flow patterns presented here.

\section{Computational Fluid Dynamics}

\section{A. Background}

In its broadest definition, CFD uses numerical computation methods to solve the governing equations of fluid motion and the energy equation for a given geometry with a computer. First, a geometric model of the ETD is constructed in a computer aided drafting (CAD) software application. This model is then discretized into small computational zones, a process known as meshing. An example of a meshed computational model is shown in Figure 1. The meshing of 2-dimensional models is fairly straightforward; however 3-dimensional models often require additional effort along with significant processor demands. Once a model has been discretized, boundary conditions are assigned to each section of the model and are used to simulate the actual conditions set by the instrument. Examples of common boundary conditions include velocity inlet, pressure inlet, pressure outlet, temperature profile, and wall.

With all boundary conditions established, a number of additional parameters and solving schemes can be selected, all of which are beyond the scope of this paper. Initial conditions are assigned to the model and are used to help speed the convergence of the computation. The computation is an iterative process that solves the governing equations for flow and energy in each discretized zone or cell. Depending on the complexity of the model and the computer resources available, numerical convergence can take anywhere from minutes to days. The results of the simulation can be viewed and manipulated with postprocessing software once the simulation has converged to a solution.

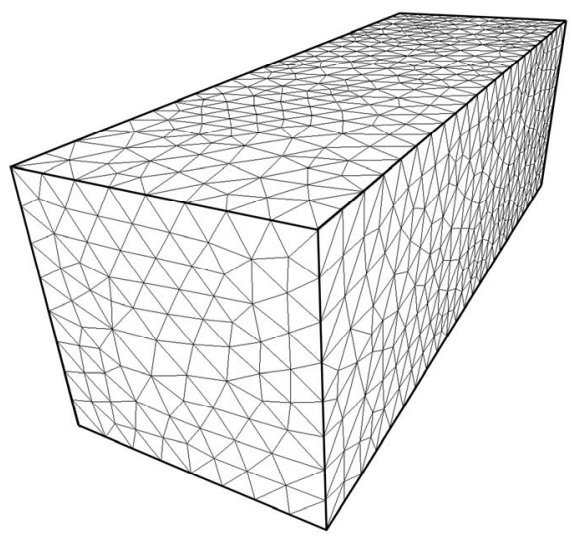

Figure 1 - Example of a meshed computational model for a rectangular box. The domain is discretized into small computational volumes where the governing equations of fluid motion are solved by a spatial differencing algorithm.

\section{B. Computational Model 1}

The first model described here is of a generic swipe-based ETD that might use two heated parallel plates as the desorption unit. In this design, a screener would swipe the surface of an object and then place the swipe vertically into the desorption unit between the parallel plates. Air is drawn into the chemical analyzer axially through a hole in the center of the desorption unit. There is a smaller hole located in the center of the collection swipe to allow transport of vapors from both sides of the swipe to the chemical analyzer. Figure 2 shows a schematic diagram of the thermal desorption unit described here.

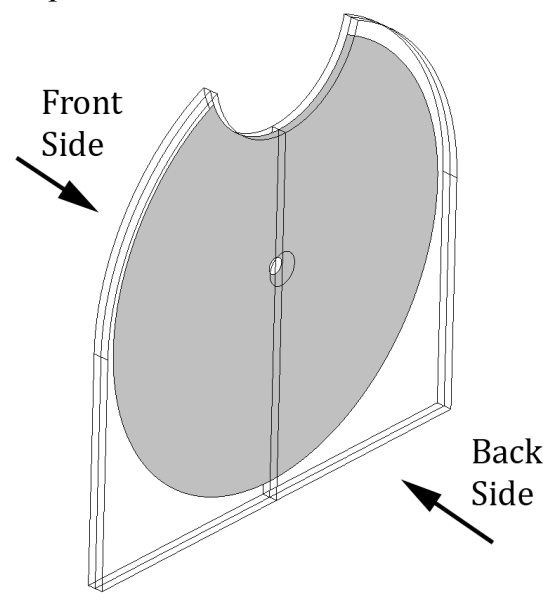

Figure 2 - Schematic diagram of the first computational model discussed here; a parallel plate thermal desorption unit. The grey circular region represents the swipe which is inserted from the top of the unit. Desorbed vapors are transported to the IMS analyzer through the hole on the back side of the domain. Air can pass into and out of all outer edges of the domain. 

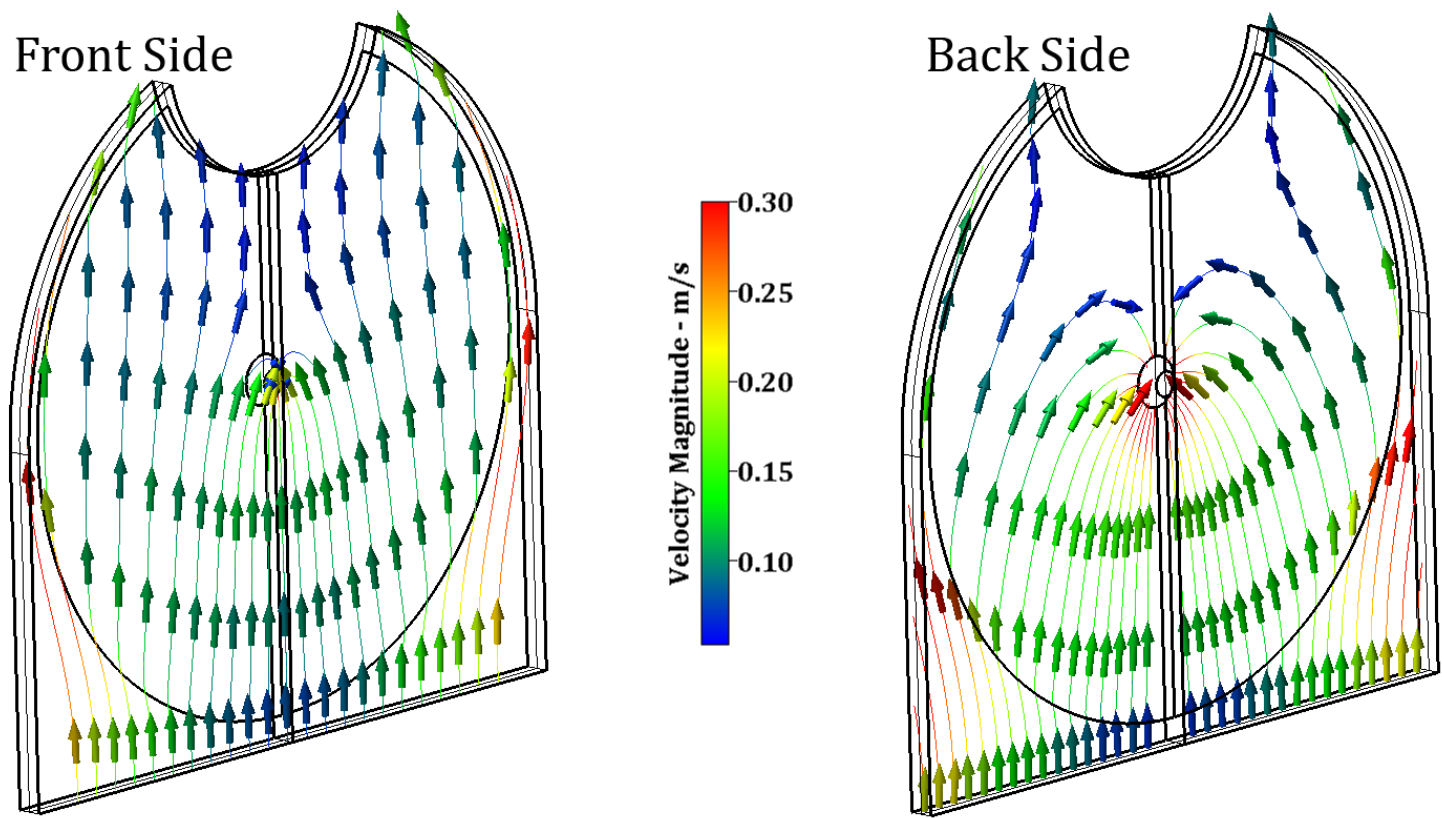

Figure 3 - Computed velocity fields on the front side and back side of the 3D thermal desorption unit. Since the desorber unit is continuously heated, a buoyant thermal plume establishes a bulk fluid motion in the vertical direction. The majority of vapor on the front side of the swipe is carried out of the domain, suggesting that the side of a swipe that has collected a sample should be inserted towards the back side and positioned downwards into the desorber unit.

The interior of the desorber is modeled as two computational domains, separated by a circular swipe. The swipe is modeled as an infinitely thin, adiabatic wall. The domain between the swipe and the front of the desorber unit is defined as the "front side" since this is the side that is closest to the security screener. The domain between the swipe and the back wall (analyzer inlet) is defined as the "back side." The parallel planar faces on either side of the swipe are modeled as walls with no slip, which are kept at a constant temperature. There is a small gap between the swipe and each of the desorber walls. The inlet to the analyzer is modeled as a velocity outlet which withdraws air from the domain at a constant flow rate. Outside air at ambient temperature is drawn into the domain through the circumferential edges which are modeled as constant pressure inlets at a pressure of $1 \mathrm{~atm}$.

Figure 3 shows the computed velocity fields for the thermal desorption unit containing a swipe. Buoyancy effects due to constant heating dominate the flow-field and produce a vertical bulk fluid motion within the domain which opposes the flow drawn into the analyzer. The back side of the domain has a larger flow rate than the domain on the front side due to its proximity to the analyzer inlet and the size difference between the inlet and the swipe holes. The majority of the flow transported to the analyzer originates from the lower half of the swipe on the back side. On the front side of the swipe, more flow escapes the desorber unit since the flow rate is significantly reduced through the swipe's central hole. In the front side swipe image in Figure
3 , note that the net direction of the velocity vectors is upward out of the domain rather than radially towards the swipe hole and analyzer inlet. This suggests that a swipe should always be placed with its collection side towards the analyzer inlet (back side) to optimal sampling efficiency.

\section{Computational Model 2}

The next model is of another possible type of thermal desorption unit and could be described as an insertion desorber. A screener would swipe the surface of an object and then insert the swipe horizontally into an enclosed heating chamber held at a constant temperature. A switch is triggered once the swipe is inserted and the analysis begins. The geometry of this heating chamber is best described in Figure 4, which also shows the computed velocity field. Note that this is a semi-enclosed system where spillage outside of the unit is minimized.

In this model, air is drawn into the IMS analyzer from two holes, one on top and one on bottom, located in the center of the chamber domain. Vapor-rich air is transported through these holes, and then turns 90 degrees down a small channel which discharges into a larger chamber (illustrated as the grey cylinder labeled "Outlet to IMS" in Figure 4).

Two notable design elements in this thermal desorption unit that significantly improve its capability are the small slits 
near the front of the swipe chamber (shown by arrows). The slits allow fresh air from outside of the desorption chamber to enter the domain and transport vapors that may be present near the front tip of the swipe. Without these slits, the front of the swipe would effectively be a dead-zone for the transport of vapors to the IMS since all make-up air would enter the domain from the swipe insertion point only.

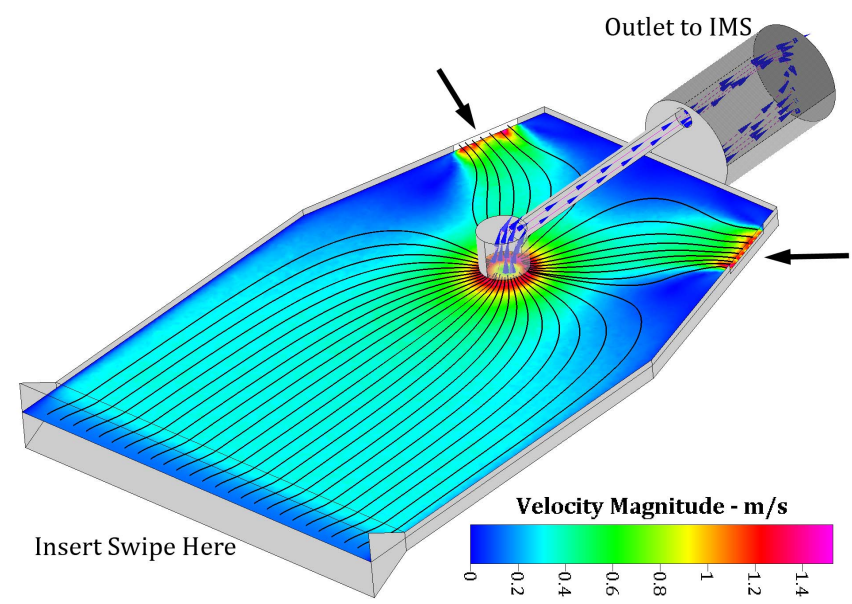

Figure 4 - Computational results of an enclosed thermal desorption chamber. Color contours represent velocity magnitude. Streamlines of the velocity field are shown as black lines. Air enters the chamber through the swipe insertion point, along with two slits located near the tip of the swipe (shown by arrows). These slits allow fresh air from outside of the desorption chamber to enter the domain and aid in the transport of vapors from the upper portion of the swipe to the center transport tube (shown as blue arrows) and then to the IMS.

\section{Computational Model 3}

The next model discussed here could be used in a swipebased ETD where the thermal desorption unit is a completely closed system. A circular swipe is introduced into a desorber and sandwiched between a heating element and a conical transfer tube that leads to an IMS. The carrier gas enters the domain below the swipe by first entering near the heating element and then forming a circular sheath of air that passes through the circumference of the swipe at its edges. The porous swipe is rapidly heated once in contact with the heating element and conveys a carrier gas through itself, transporting any desorbed vapors along with it. Results are shown in Figure 5.

The colored lines in Figure 5 are known as streamtubes. These streamtubes illustrate the velocity field and give information about how the flow inside the desorber unit moves. Air enters the domain through the green tube, impinges and circulates around the heating element (colored light-pink), and then rises to pass through the swipe.
Streamtubes above the heating element illustrate the uniformity of the flow once it has passed through the porous swipe. Vapors are accelerated as the conical tube constricts to a smaller diameter and then turn 90 degrees and exit to the IMS analyzer.

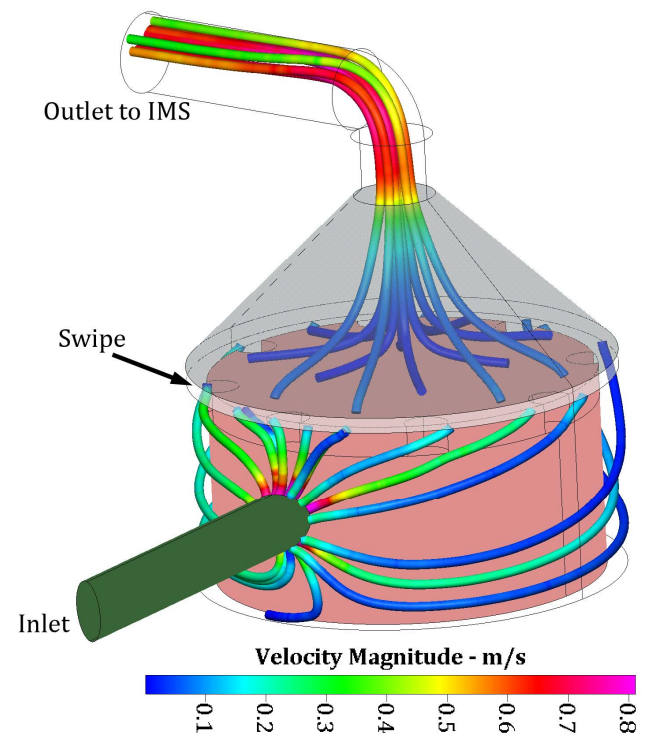

Figure 5 - Computational model of a swipe-based ETD with a fully-enclosed thermal desorption unit. Carrier gas enters the unit and circulates around the heating element (colored light-pink) before it passes through the porous circular swipe. A conical transfer tube focuses the flow, along with any desorbed vapors, to a 90 degree elbow before it exits to the IMS analyzer.

This design maximizes the transport of vapors desorbed from the surface of the swipe because it is a closed system. However, the area of the swipe that is actually sampled represents approximately $40 \%$ of the total area of the swipe. The portion of the swipe that is not sampled lies outside of the heating element and transfer tube in this closed system. Users of this system should ensure that the swipe collects material as close to the center of the swipe as possible during screening procedures. Any material collected on the outer edges of the swipe (not modeled here) will not enter the desorber unit and will not be analyzed.

\section{E. Computational Model 4}

The next conceptual design modeled here is of a swipebased ETD with dual-mode analysis capabilities. Detection of both explosives and narcotics is accomplished by using two separate IMS units in a single instrument. Similar to the design described in Computational Model 3, a circular swipe is introduced into a desorber and sandwiched between a heating element and a conical transfer tube that leads to an IMS. Here, the heating element is positioned on top of a cylindrical stage. The volume under the heating element is a 
void chamber where the carrier gas enters vertically from the bottom. The gas swirls inside this chamber and then flows around the outer edges of the heating element and through the porous swipe. After the swipe, the carrier gas converges in a conical nozzle and then splits equally between two outlet tubes. Each outlet tube feeds desorbed vapors to one of the two IMS analyzers. Results are shown in Figure 6.

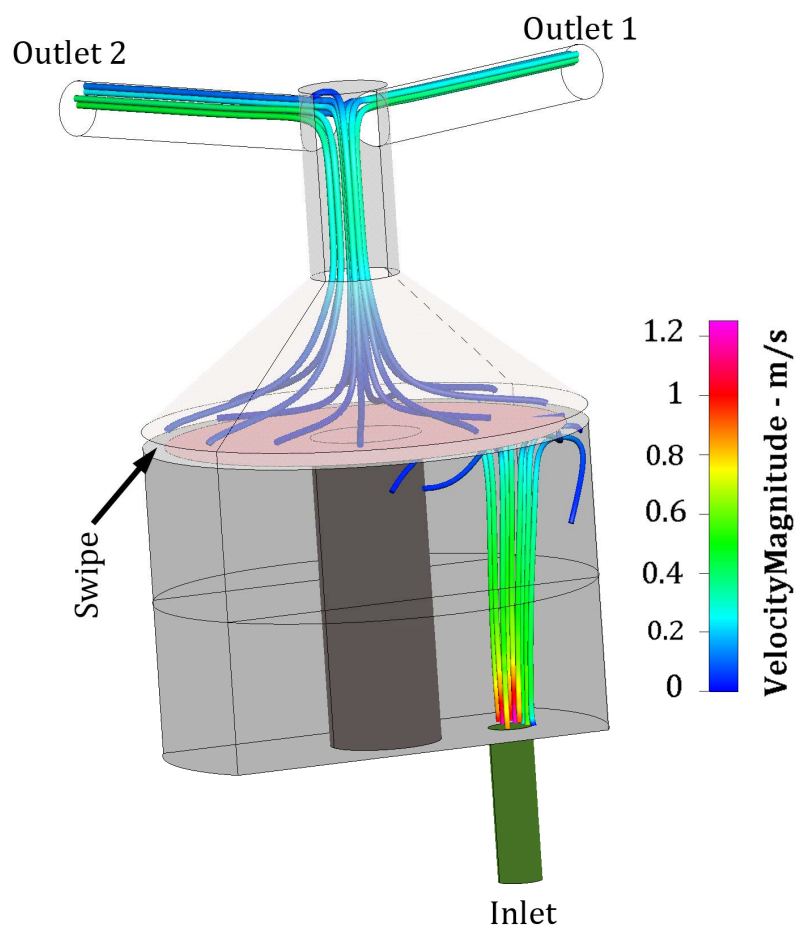

Figure 6 - Another computational model of a swipe-based ETD with a fully-enclosed thermal desorption unit. Carrier gas enters a void chamber and impinges on the heating element (colored light-pink). The gas then circulates within the void chamber before permeating through the porous swipe at a constant flow rate. Desorbed vapors from the swipe are carried through a conical nozzle before being equally split between the two outlets which lead to each IMS analyzer.

Carrier gas emanating from the inlet tube is seen entering the void chamber and impinging upon the heating element (colored light-pink). This suggests that the flow through the porous swipe may be uneven and favor the side nearest the inlet tube. However, the swipe acts as a resistance to the flow and stabilizes the flow rate through itself. This is evidenced by the streamtubes above the swipe which have a regular flow pattern that begins at the surface of the swipe and travels through the conical transfer tube. The simulation indicates that the flow to each outlet is split evenly, showing that each IMS receives that same fraction of vaporized sample during the analysis.

\section{F. Computational Model 5}

The next model is a generic concept for a sampling unit of a vapor sniffing ETD which is mounted on the snout of the instrument. The chemical analyzer on this instrument uses a membrane to increase ionization efficiency. The computational results for this vapor sampling unit are shown in Figure 7.

The sampling inlet on this sniffer is the nose of the instrument that uses two vacuum pumps to draw air into a hole in the center of the snout. Air enters the domain at the center hole (colored black) and leaves the domain through two pumps (colored pink). The red-colored disk in the center represents the membrane that is used in this design.

Computational modeling of this particular sampling inlet reveals several interesting fluid dynamics. First, air enters axially through the inlet (black) and immediately impinges upon the membrane (red). After impingement, a small fraction of the flow travels across the membrane and exits the domain through the pump outlets (pink). The majority of the flow, however, remains near the membrane before being evacuated. This design establishes recirculation-wells that allow sampled air to be passed over the membrane several times before exiting the domain. These recirculation-wells can be visualized by the streamtubes that are twisting and curling near the membrane. The recirculation-wells maximize the uptake of explosive vapors through the membrane during sampling. 


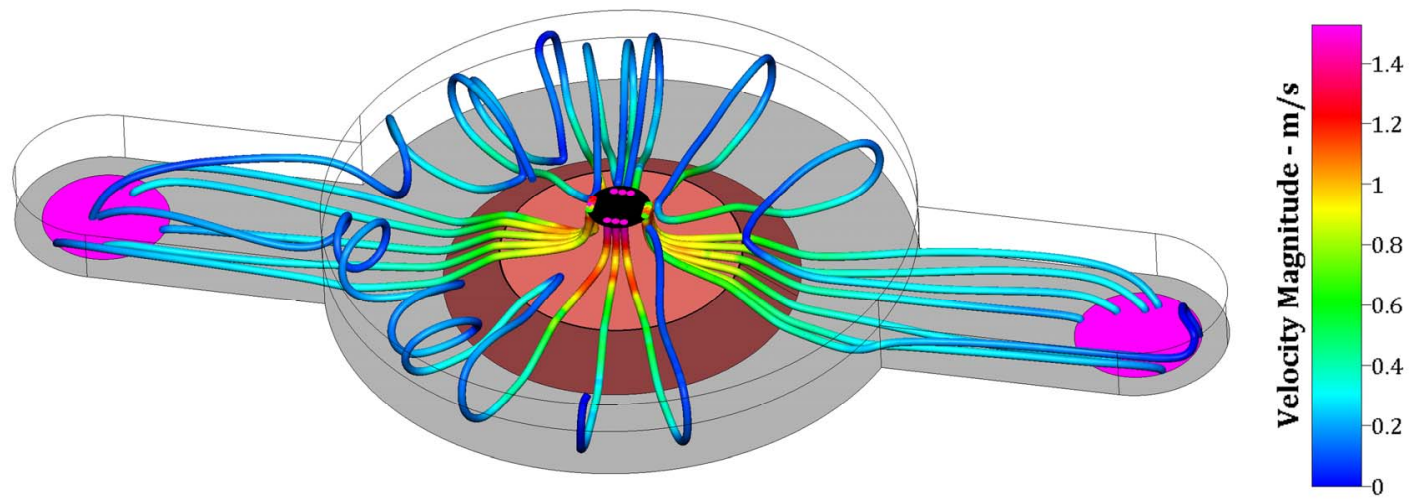

Figure 7 - Computational velocity streamline results for a vapor sampling unit. Air is drawn into the unit through the black hole in the center and immediately impinges on the membrane (colored red). Recirculation-wells are established which pass the sample over the membrane several times before exiting through two holes (colored pink) to dual suction pumps.

\section{G. Computational Model 6}

The final model shown here could be used as the sampling unit for a vapor sniffing ETD. The sampling inlet in this design uses a unique approach for vapor sampling. Stationary rotor blades are positioned annularly around a central sampling inlet that leads to the detector. A small blower forces air through the rotor blades which creates a vortex that is projected from the unit. Meanwhile, air is being drawn into a sampling hole in the center of the unit and then to a chemical analyzer. The outer envelope of this vortex can be seen in Figure $8 \mathrm{~b}$ as the light-grey funnel emanating from the unit and streamlines are shown as black lines in Figure 8a. This vortex creates a low pressure zone in its center, allowing the inlet to effectively sample vapors at distances upwards of tens of centimeters.
Schlieren imaging [6] is used here for computational verification. The schlieren optical technique allows one to visualize the refractive index, i.e. density gradient, of transparent media. With this technique, density gradients of vapors are readily visualized as they are sampled by a vapor detector. Shown in Figure 9, the sampling unit is placed in the schlieren beam as it samples acetone vapors. The image shows two red arrows representing the downward vortex emanating from the aerodynamic sampling unit, while the white arrow shows the direction of acetone vapor being vectored into the chemical analyzer.

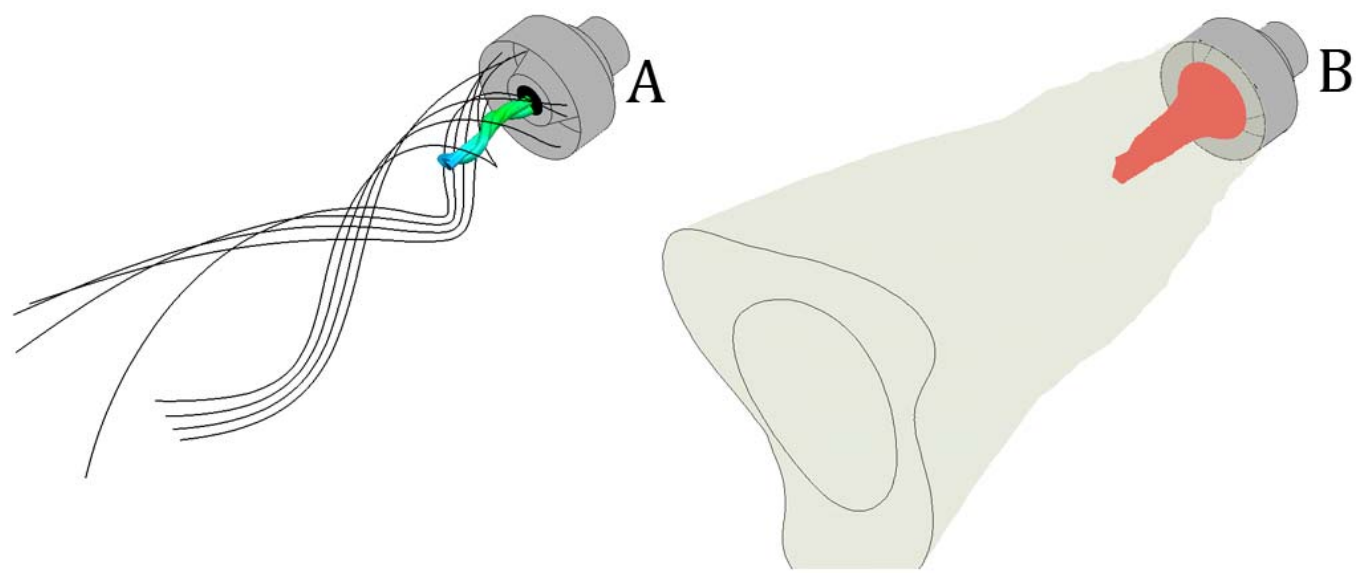

Figure 8 - Computational model of a sampling inlet. The grey circular component represents the aerodynamic sampling unit, made of stationary rotor blades located circumferentially around central a sampling inlet. In A, black streamlines illustrate the vortical motion created by air pushed through the rotor blades while colored streamtubes represent the effective aerodynamic reach. In B, the emanating vortex is shown as the tan-colored envelope while the aerodynamic reach of the inlet is shown in red. 


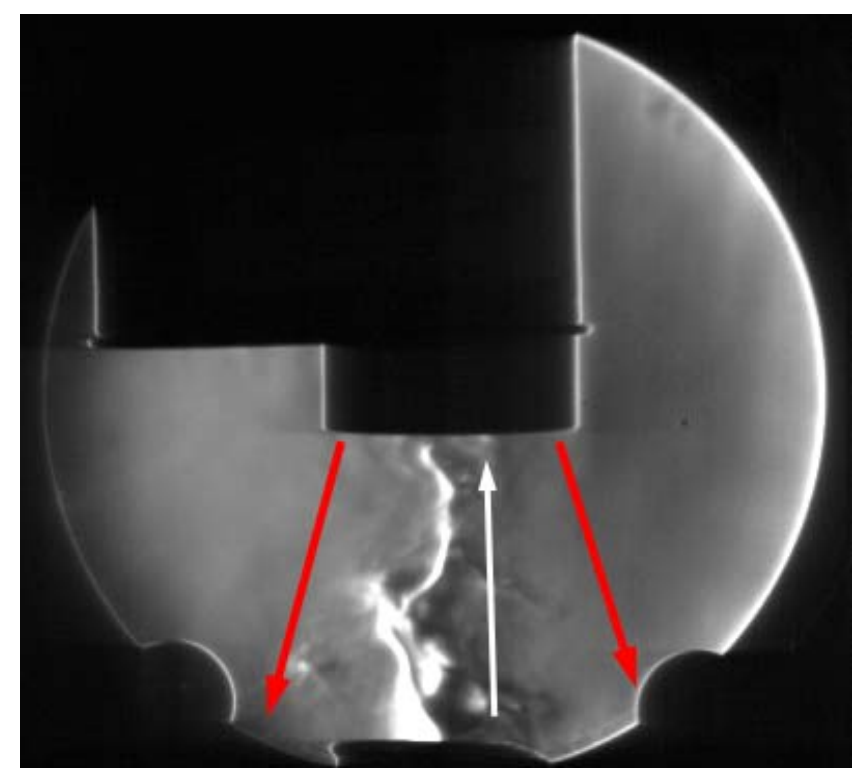

Figure 9 - Experimental flow visualization demonstrating the sampling of acetone vapors in a schlieren optical system. Red arrows represent the vortical flow protruding from the snout of the unit. The white arrow illustrates the transport of acetone vapors, seen as light and dark contrasts, from the source to the sampling inlet.

\section{CONCLUSIONS}

Computational Fluid Dynamics has revealed the flowfield characteristics inside several thermal desorption units and sampling inlets that have geometries representative of what could be used by explosive trace detectors. Understanding the underlying physics of the flowfields inside these instruments may help researchers and manufacturers design new sampling strategies, improve functionality of existing designs, develop standard test materials for instrument verification, and develop next-generation instruments that optimize the internal geometry from a fluid mechanics perspective. Future work will focus on verifying the computational results with traditional flow visualization techniques such as laser light scattering, particle image velocimetry, and schlieren imaging.

\section{ACKNOWLEDGEMENT}

The Department of Homeland Security Science and Technology Directorate sponsored this work under an interagency agreement with the National Institute of Standards and Technology

\section{REFERENCES}

[1] D. S. Moore, Instrumentation for trace detection of high explosives, Rev Sci Instrum 75 (8), 2499-2512, 2004.

[2] J. R. Verkouteren, Particle characteristics of trace high explosives: RDX and PETN, J Forensic Sci 52 (2), 335-340, 2007.

[3] R. G. Ewing, D. A. Atkinson, G. A. Eiceman, and G. J. Ewing, A critical review of ion mobility spectrometry for the detection of explosives and explosive related compounds, Talanta 54 (3), 515-529, 2001.

[4] G. S. Settles, Fluid mechanics and homeland security, Annu Rev Fluid Mech 38, 87-110, 2006.

[5] R. M. Verkouteren, G. Gillen, J. R. Verkouteren, R. A. Fletcher, and W. J. Smith, NIST program to support T\&E of trace explosives detection, J Test Eval 28 (3), 16-18, 2007.

[6] G.S. Settles, Schlieren and shadowgraph techniques: Visualizing phenomena in transparent media, Springer-Verlag, Berlin, 2001. 